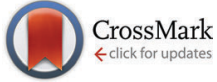

Cite this: Phys. Chem. Chem. Phys., 2015, 17, 16015

Received 24th March 2015,

DOI: $10.1039 / c 5 c p 01696 g$

www.rsc.org/pccp Accepted 8th May 2015

\section{Complexation induced fluorescence and acid-base properties of dapoxyl dye with $\gamma$-cyclodextrin: a drug-binding application using displacement assays $\uparrow$}

\begin{abstract}
Kaushik Pal, \$Suman Mallick $\ddagger$ and Apurba L. Koner*
Host-guest complexation of dapoxyl sodium sulphonate (DSS), an intramolecular charge transfer dye with water-soluble and non-toxic macrocycle $\gamma$-cyclodextrin $(\gamma$-CD), has been investigated in a wide $\mathrm{pH}$ range. Steady-state absorption, fluorescence and time-resolved fluorescence measurements confirm the positioning of DSS into the hydrophobic cavity of $\gamma$-CD. A large fluorescence enhancement ca. 30 times, due to $1: 2$ complex formation and host-assisted guest-protonation have been utilised for developing a method for the utilisation of CD based drug-delivery applications. A simple fluorescence-displacement based approach is implemented at physiological pH for the assessment of binding strength of pharmaceutically useful small drug molecules (ibuprofen, paracetamol, methyl salicylate, salicylic acid, aspirin, and piroxicam) and six important antibiotic drugs (resazurin, thiamphenicol, chloramphenicol, ampicillin, kanamycin, and sorbic acid) with $\gamma-C D$.
\end{abstract}

\section{Introduction}

Formulation of water-insoluble drugs with the help of watersoluble and non-toxic macrocyclic host molecules has received substantial interest. ${ }^{1-10}$ The encapsulation of small drug molecules by water-soluble macrocyclic hosts due to non-covalent interactions can reinforce bioavailability and solubility by modulating their physical and chemical properties. ${ }^{5-8}$ The encapsulation of drug molecules inside hydrophobic cavities leads to modulations of their solution properties, and this triggers the activation of the drug from its proactive form to its active state. ${ }^{11-14}$ Supramolecular drug-macrocycle complexes have already been shown to have great promise for their usefulness in targeted drug delivery applications. ${ }^{15-17}$

Naturally occurring, non-toxic and water-soluble cyclodextrins (CDs) can encapsulate drugs in their hydrophobic cavity. CDs are a family of cyclic compounds composed of sugar units connected by 1,4-glycosidic linkages, and depending on the number of units in the cyclic structure, they are denoted with different names. Alpha-, beta- and gamma-cyclodextrin consist of six, seven and eight sugar units, respectively. ${ }^{18} \mathrm{CD}$ cavities provide an access to a "new phase of matter" to the encapsulated guest molecule,

Department of Chemistry, Indian Institute of Science Education and Research Bhopal, Bhopal, India. E-mail: akoner@iiserb.ac.in; Fax: +91 7556692 392; Tel: +917556692376

$\dagger$ Electronic supplementary information (ESI) available. See DOI: 10.1039/ c5cp01696g

‡ Authors contributed equally. which is very different from the exterior environment. ${ }^{19-21}$ These unique cavities can modulate the solution and optical properties of the encapsulated fluorescent guest. Due to noncovalent interactions in the complex, the physical and chemical properties of the encapsulated molecules can be modulated to provide protection against photochemical and thermal decomposition, oxidation or hydrolysis. ${ }^{22,23}$ The larger cavity size together with higher water-solubility compared to its homologues, ${ }^{24,25}$ prompted us to select $\gamma$-CD (see Fig. 1a), which is sufficiently spacious to accommodate moderate-to-large drug molecules for assessing its utility for drug-delivery applications. The same drug-delivery application is not always possible using lower homologues of $\gamma$-CD (i.e. $\alpha$-CD and $\beta$-CD) because their

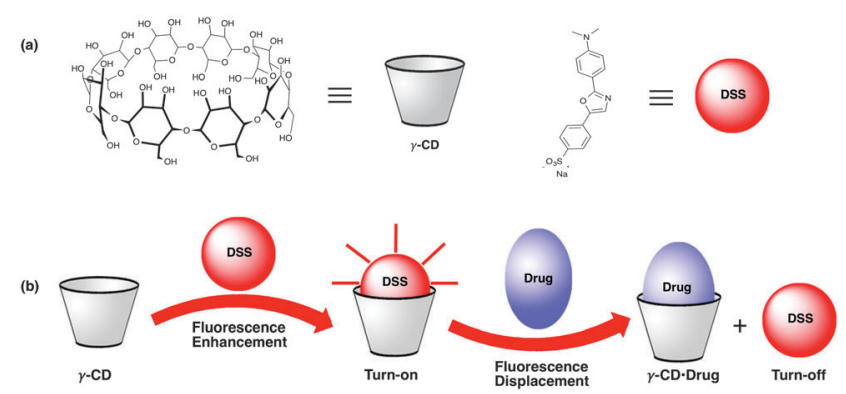

Fig. 1 (a) Structure of $\gamma$-cyclodextrin and DSS, (b) schematic presentation of complexation-induced fluorescence enhancement of DSS and the switch-off fluorescence displacement principle based on the displacement principle to determine the drug binding constant. 
cavity volume is not enough to accommodate drug molecules with larger size, and in most cases results in partial encapsulation. Consequently, such partial encapsulation often provides an insignificant modulation of properties and is not suitable for the aforementioned applications. Furthermore, the water-solubility of $\gamma$-CD is 10 times higher than that of $\beta$-CD and this allows better drug solubility upon encapsulation.

Generally, in pharmaceutical industries, water-insoluble drugs are formulated using hydrophilic polymers, small molecule additives or via macrocyclic encapsulation. ${ }^{26,27}$ CDs are a well-known host molecule for solubilising drugs and there are quite a few CD formulated drugs already available commercially. ${ }^{28}$ Spectrophotometry, thermogravimetric analysis (TGA), XRD, differential scanning calorimetry (DSC) and NMR are commonly used methods to validate the suitability of CDs as a co-solute for drug molecules. ${ }^{29}$ However, these established methods are time consuming and need quite a significant amount of drugs and solubiliser for evaluation.

Fluorescence-based indicator displacement assays ${ }^{30-32}$ can be an alternative and easy-to-implement method to test the suitability of $\mathrm{CD}$ in general as a solubiliser for water-insoluble drugs. In a displacement assay, a competitive analyte is introduced to a solution containing a host-dye complex and upon the displacement of the dye from the receptor a significant change in the optical signal occurs, which in turn allows one to evaluate the analyte-binding ability of the receptor. To develop a displacement assay, we need a fluorescent molecule with differential optical properties in its complex and free forms. An environmentally sensitive fluorophore would be an ideal choice for such a method. Considering this fact, dapoxyl sodium sulphonate (DSS, Fig. 1a) was selected as the fluorescent dye, which is an intramolecular charge transfer (ICT) dye having both electron rich and deficient groups. ${ }^{20,33-36}$ Immediately after the excitation of an ICT dye, it reached the Franck-Condon state or locally excited state (LE) and upon solvation, a relaxed ICT state was achieved. Due to the large excited state dipole moment, the solvent relaxation exhibits a red-shifted fluorescence spectrum as the polarity of the solvent increases. ${ }^{37,38}$ Likewise, DSS exhibits solvent polarity dependent fluorescence properties. ${ }^{33,34,39,40}$ A recent study was performed by us to validate the solvent polarity and $\mathrm{pH}$ dependent photophysical properties of DSS. ${ }^{40}$ A $1: 1$ complex formation of DSS with $\alpha$-CD and $\beta$-CD cavities results in a large fluorescence enhancement along with a blue-shift in the emission maxima. ${ }^{40}$ This finding prompts us to further investigate the binding of DSS with larger cavity $\gamma$-CD with the anticipation of discovering novel photophysical properties of DSS, which can be implemented to develop a displacement assay for assessing the binding of sparingly-soluble drugs with $\gamma$-CD (Fig. 1b). In general, it is very difficult to find a suitable fluorescent dye that can form a 1:1 host-guest complex with spacious $\gamma$-CD cavity and exhibits novel photophysical properties for displacement applications. ${ }^{5}$ Moreover, in most cases, large guest molecules result in $2: 1$ complexes, which make the appropriate analysis of the displacement assay more challenging. ${ }^{41}$ Positively charged surfactants and DNA have been used as competitors for the fluorescent-based displacement method using $\beta$-CD. ${ }^{42,43}$ Ueno and co-workers reported a fluorescentbased displacement method by implementing a covalently attached dye with a CD surface. ${ }^{44}$ Recently, an NMR-based displacement method has been reported to test the drugbinding ability of $\mathrm{CD}^{45}$ A highly sensitive method such as a fluorescence-based turn-on or turn-off technique will be well suited for such an application. To the best of our knowledge, there is currently no report on fluorescence-based displacement assays using CD to validate drug binding.

Herein, we report the $\mathrm{pH}$-dependent fluorescence properties of DSS upon its 1:2 complex formation with $\gamma$-CD. Furthermore, the modulated properties have been used to foster a novel, fluorescencebased and easy-to-perform turn-off fluorescence displacement method for assessing the drug-binding ability.

\section{Experimental section}

\subsection{Materials}

DSS was purchased from Invitrogen (USA); $\gamma$-cyclodextrin $(\gamma$-CD) and $\mathrm{Na}_{2} \mathrm{HPO}_{4}$ were purchased from Spectrochem (India); paracetamol, $\mathrm{NaOH}, \mathrm{NaCl}, \mathrm{KCl}$ and $\mathrm{HCl}$ were purchased from SDfine (India); salicylic acid was obtained from Merck (India); methyl salicylate was obtained from Loba Chemie (India); ibuprofen was obtained from Alfa-Aesar (India); piroxicam, aspirin, resazurin, sorbic acid, and thiamphenicol were purchased from Sigma-Aldrich (India), chloramphenicol, ampicillin, kanamycin were purchased from BR Biochem Life Science (India). $\mathrm{KH}_{2} \mathrm{PO}_{4}$ was obtained from $\mathrm{CDH}$ chemical (India). All the chemicals were used as they were received without performing any further purification.

\subsection{Steady-state absorption and fluorescence measurements}

Steady-state absorption measurements were performed on a Shimadzu UV-Spectrophotometer 1800 (UV probe 2.42 software) using a $1 \mathrm{~cm}$ path length quartz cuvette. All steady-state fluorescence measurements were carried out using HORIBA Jobin Yvon Fluoromax-4 fluorimeter with the Origin 8 software provided with the instrument. A $5 \mu \mathrm{M}$ solution of DSS was taken for all the measurements to maintain the absorption value constant so that we can avoid the inner filter effect. The experiment was carried out using Milli-Q grade water using Millipore water purification set up with the resistivity of $18.2 \mathrm{M} \Omega \mathrm{cm}$ at $298 \mathrm{~K}$. The $\mathrm{pH}$ of aqueous solutions was adjusted using $\mathrm{HCl}$ and $\mathrm{NaOH}$ solutions. All the $\gamma$-CD stock solutions were prepared in the same DSS solution and the $\mathrm{pH}$ was adjusted appropriately. A displacement assay was performed at physiological $\mathrm{pH}$ ca. 7.4 with $\gamma$-CD with different concentrations of drugs varying from 0 to $100 \mu \mathrm{M}$. Fluorescence spectra were recorded using a $1 \mathrm{~cm}$ path length quartz cuvette from 355 to $680 \mathrm{~nm}$ by exciting at $340 \mathrm{~nm}$ and keeping both the excitation and emission slit at $2 \mathrm{~nm}$. All the experiments were carried out at ambient temperature (298 K).

\subsection{Time-resolved measurements}

Time-resolved fluorescence measurements were performed using a Hamamatsu MCP photomultiplier (R-3809U-50). The time-correlated single photon counting (TCSPC) setup consists 
of an Ortec 9327 pico-timing amplifier and a pulse diode laser (NanoLED, N-375) for excitation $\left(\lambda_{\mathrm{ex}}=375 \mathrm{~nm}\right)$ with fwhm $=$ $\sim 167$ ps and a setup target of 10000 counts. The instrument response function (IRF) was measured before and after the fluorescence lifetime measurements using a dilute suspension of Ludox (purchased from Sigma) colloidal silica. The emission polarizer was positioned at the magic angle $\left(54.7^{\circ}\right)$ with respect to the excitation polarizer. Single and multi-exponential fitting functions were employed by an iterative deconvolution method using the supplied software DAS v6.2. The general form of the fitting function can be given as follows:

$$
\frac{I(t)}{I(0)}=\sum a_{i} \exp \left(-t / \tau_{i}\right)
$$

where $I(t)$ and $I(0)$ are the fluorescence intensity at time $t$ and 0 , respectively, $t$ is time and $a_{i}$ and $\tau_{\mathrm{t}}$ are the contributing amplitude and its corresponding lifetime. The quality of the fitted data was judged from the reduced chi-squared value $\left(\chi^{2}\right)$, which is calculated using the IBH software provided with the instrument. All the measurements were carried out at ambient temperature $(298 \mathrm{~K})$.

\subsection{Drug binding experiment}

A displacement assay was performed in an aqueous solution at $\mathrm{pH}$ ca. 7.4 containing $5 \mu \mathrm{M}$ DSS and $5 \mathrm{mM} \gamma-\mathrm{CD}$ in a $1 \mathrm{ml}$ fluorescence cuvette. Then, the fluorescence intensity was measured at $500 \mathrm{~nm}$ upon excitation at $337 \mathrm{~nm}$ with the subsequent addition of the $\mathrm{pH}$ adjusted concentrated drug solution in the same $\gamma$-CD.(DSS) solution to avoid dilution and changes in $\mathrm{pH}$. The binding constants of $\gamma$-CD.drug were calculated using $1: 2$ and $1: 1$ binding equations for the small-molecule drugs and for the antibiotic, respectively. The concentration of the $\gamma$-CD.DSS complex was calculated from the binding constant of the $\gamma$-CD-DSS complex at $\mathrm{pH}$ 7.4.

\section{5. $\quad \mathrm{pH}$ titration, binding titration and their analysis}

The pH-dependent absorbance of the DSS dye was measured to determine its $\mathrm{p} K_{\mathrm{a}}$ value using a two-state model ${ }^{40,46}$ (by considering the protonated and non-protonated dye) and in the case of the $\gamma$-CD.DSS complex, a four-state model- ${ }^{40,46}$ was used to determine the $\mathrm{p} K_{\mathrm{a}}$ value in the complex state. The $\mathrm{pH}$ dependent binding constants of the DSS dye with $\gamma$-CD was fitted with a $1: 2$ binding equation using a nonlinear fitting procedure of the Pro Fit 6.2.9 software, as reported earlier by Nau and co-workers. ${ }^{47}$ In all the measurements, the error was calculated after fitting the experimental data using an appropriate equation with the help of Pro Fit 6.2.9 software. The software provides the goodness of fit as the difference of the mean value as an error after fitting and it was within $\pm 10 \%$.

\subsection{Computational study of complexation}

To investigate the mode of complexation between $\gamma$-CD and DSS, we performed a computational study. In this regard, we employed density functional theory (DFT) based molecular module DMol3 in the Material Studio 6.1 programme package. ${ }^{48}$ The Perdew-Wang generalised-gradient approximation functional (PWC) was used for all the geometry optimisations. ${ }^{49}$ To make our computational study consistent, we used the conductor-like screening model (COSMO) for applying solvent effect on the complexation in water. The electrostatic energy during the optimisation is given as follows:

$$
E_{\text {elec }}^{\text {COSMO }}=-\frac{1}{2}\langle Z|D| Z\rangle-\langle\rho|D| Z\rangle-\langle\rho|D| \tilde{\rho}\rangle+\frac{1}{2}\langle\tilde{\rho}|D| \tilde{\rho}\rangle
$$

where $Z$ is the nuclear charge, $D$ is the dielectric operator, $\rho$ is the density and $\tilde{\rho}$ is the auxiliary density; here, auxiliary density is introduced to solve the Poisson equation for the electrostatic potential of the solute.

\section{Results and discussion}

The present study deals with the $\mathrm{pH}$ dependent complexation of DSS with $\gamma$-CD and the determination of the drug binding ability of $\gamma$-CD. DSS binding with $\gamma$-CD causes a large fluorescence enhancement and a shift in the acid dissociation constant and this helped us to design a turn-off indicator displacement assay. UV-Vis and fluorescence titration of DSS with $\gamma$-CD were performed at different $\mathrm{pH}$ values and the complexation-induced fluorescence change was monitored to calculate the binding constant of DSS with $\gamma$-CD. A Job's plot at pH 7.0, obtained by monitoring the fluorescence intensity changes, confirms a $1: 2$ complexation between $\gamma$-CD and DSS. Differential binding affinity at different $\mathrm{pH}$ values indicated a shift in the acid dissociation constant and a negative shift of one unit was measured by the $\mathrm{pH}$ titration of DSS and its $\gamma$-CD.(DSS $)_{2}$ complex. Furthermore, binding of small molecules and antibiotic drugs with $\gamma$-CD were estimated using the turn-off displacement principle. The results and discussion section is subdivided into subsections detailing DSS binding to $\gamma$-CD, host-induced $\mathrm{p} K_{\mathrm{a}}$ shift, lifetime, anisotropy properties and drug-binding study using the displacement principle.

\section{1. $\quad$ pH-dependent binding studies of DSS with $\gamma$-CD using absorption and fluorescence spectroscopy}

Host-guest titration at pH 7.0 using $5 \mu \mathrm{M}$ DSS was performed by the successive addition of a concentrated stock solution of

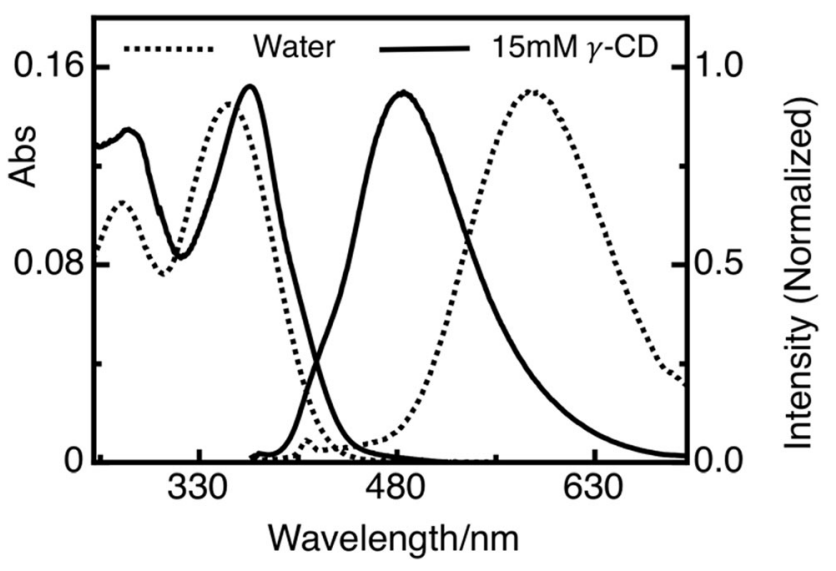

Fig. 2 Absorption and fluorescence spectra of DSS in water and with $15 \mathrm{mM} \gamma$-CD at ca. $\mathrm{pH} 7.0$. 

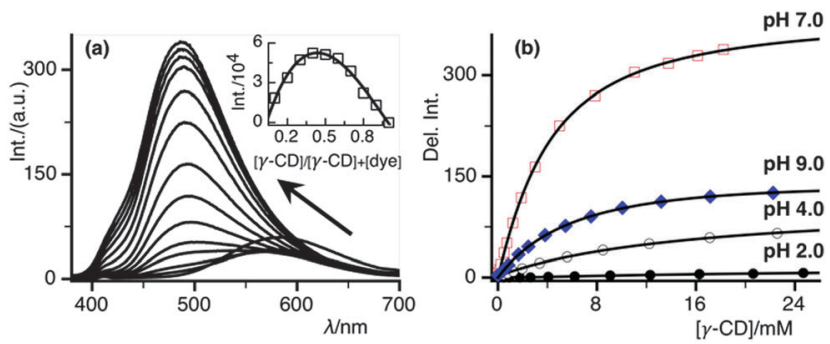

Fig. 3 (a) Fluorescence titration of DSS with $\gamma-\mathrm{CD}$ at $\mathrm{pH}$ 7.0, showing a shift in the emission maxima with a gradual increase in the fluorescence intensity, inset shows Job's plot and it confirms the 1:2 complexation of $\gamma$-CD and DSS at pH 7.0, (b) pH-dependent fluorescence enhancement of DSS with $\gamma-C D$ monitored at $480 \mathrm{~nm}$, data fitted with 1:2 binding model.

Table 1 Photophysical properties of DSS in water and in $\gamma$-CD complex

\begin{tabular}{llllllll}
\hline & $\begin{array}{l}\lambda_{\mathrm{abs}} \\
(\mathrm{nm})\end{array}$ & $\begin{array}{l}\lambda_{\mathrm{em}} \\
(\mathrm{nm})\end{array}$ & $\begin{array}{l}\text { Stokes shift } \\
\left(\mathrm{cm}^{-1}\right)\end{array}$ & $\mathrm{QY}^{b}$ & $\begin{array}{l}\tau^{c, d} \\
(\mathrm{~ns})\end{array}$ & $\begin{array}{l}k_{\mathrm{r}} \times 10^{8} \\
\left(\mathrm{~s}^{-1}\right)\end{array}$ & $\begin{array}{l}k_{\mathrm{nr}}^{e} \times 10^{8} \\
\left(\mathrm{~s}^{-1}\right)\end{array}$ \\
\hline Wedium & 342 & $383^{a}, 582$ & 12058 & 0.19 & 1.9 & 1.00 & 4.26 \\
$\gamma$-CD & 369 & 488 & 6608 & 0.68 & 6.1 & 1.11 & 0.52
\end{tabular}

${ }^{a}$ Obtained at pH 2.0 and 4.0 due to LE state. ${ }^{b} \mathrm{QY}$ was calculated using 8-anilinonapthalene-1-sulphonic acid in methanol $(\mathrm{QY}=0.21)$ as a standard. ${ }^{51 c}$ Average fluorescence lifetime. ${ }^{d}$ Lifetime measured at $\mathrm{pH} 7.0$ and lifetime of DSS in other $\mathrm{pH}$ with and without the $\gamma$-CD complex are given in Table $2 .{ }^{e}$ Non-radiative rates were calculated from the formula $k_{\mathrm{nr}}=\left(1-\phi_{\mathrm{f}}\right) / \tau_{\mathrm{f}}$.

$\gamma$-CD in the same concentration as DSS. The titrations were monitored by both absorption and fluorescence spectroscopy. The absorption spectrum of DSS was characterised by a broad non-structured band spanning in the region between 300 and $480 \mathrm{~nm}$ (Fig. 2). Similarly, the emission spectrum has a broad range of 350-650 $\mathrm{nm}$. A significant red shift of $c a .27 \mathrm{~nm}$ in the absorption spectra was observed apparently due to $\mathrm{H}$-bonding interactions in the mediated complexation ${ }^{50}$ of DSS and $\gamma$-CD. The emission maximum of DSS has a considerable blue shift from 582 to $488 \mathrm{~nm}$ and a large fluorescence enhancement upon complexation with $\gamma$-CD (Fig. 3a). The FWHM of both the absorption and fluorescence spectra of DSS in the presence of $\gamma$-CD become narrower compared to the same in water due to its positioning in the hydrophobic environment and higherorder complex formation.

The complexation-induced photophysical properties of DSS from the steady-state and time-resolved measurements at pH 7.0 are summarised in Table 1. Upon $\gamma$-CD complexation, we observed 3.6 and 3.2 times increase in QY and fluorescence lifetime, respectively.

Notably, a large complexation-induced spectral shift, fluorescence enhancement and spectral feature of the $\gamma$-CD.(DSS $)_{2}$ complex at $\mathrm{pH} 7.0$ prompted us to find out the stoichiometry of the host-guest complex. The fluorescence peak observed for the complex is very different compared to the maximum of DSS in water (LE band: $383 \mathrm{~nm}$, CT band: $580 \mathrm{~nm}$ ) or of DSS in the $\beta$-CD.DSS complex (CT band: $553 \mathrm{~nm}$ ). ${ }^{40}$ The Job's plot in the inset of Fig. 3 a with a maximum around 0.40 indicates a $\gamma$-CD.(DSS $)_{2}$ complex formation.
To characterise further and evaluate the binding strength, pH-dependent fluorescence titration of DSS was performed with $\gamma$-CD and the $\mathrm{p} K_{\mathrm{a}}$ value of DSS was determine to be ca. 4.1. ${ }^{20,40}$ The protonated form of DSS $\left(\mathrm{DSSH}^{+}\right)$shows an emission maxima originating from both LE as well as from the CT state. In contrast, the spectrum only due to the CT state is visible from the non-protonated state. At a $\mathrm{pH}$ value lower than the acid dissociation constant, $\mathrm{DSSH}^{+}$binds weakly with the hydrophobic cavity of $\gamma$-CD due to the protonation of $N, N$-dimethyl aniline part of the guest molecule. On the contrary, DSS has significantly higher binding strength due to hydrophobic interaction with aromatic rings as well as hydrogen bonding interactions with sulphonate part (see Fig. S2, ESI $\dagger$ ). The binding affinities of DSS and $\gamma$-CD were calculated from the change in the fluorescence intensity of DSS upon addition of an increasing amount of $\gamma$-CD to a fixed concentration of DSS (see Fig. 3b). We also performed a computational study employing DFT based molecular module DMol3 in Material Studio 6.1 program package $^{48,49}$ to test the binding mode of the host-guest complexation. As it can be seen from Fig. S2 (ESI $\dagger$ ) that $1: 1 \gamma$-CD.DSS complex shows a significant distortion in the $\gamma$-CD structure but on the contrary, $\gamma$-CD.(DSS $)_{2}$ complex shows retention of the symmetric structure of $\gamma$-CD. pH-dependent binding constant were calculated using 1:2 fitting function ${ }^{47}$ and the values are summarised in Table 2 .

The differential binding affinity of DSS and $\mathrm{DSSH}^{+}$with $\gamma$-CD prompted us to investigate the complexation-induced shift in the acid dissociation constant of DSS. A pH titration of DSS and $\gamma$-CD.(DSS $)_{2}$ using spectrophotometry reveal a 0.9 unit negative $\mathrm{p} K_{\mathrm{a}}$ shift (Fig. 4a). The inset of Fig. 4a shows a typical $\mathrm{pH}$ titration of DSS in the presence of $15 \mathrm{mM}$ of $\gamma$-CD. The binding titration of DSS and $\gamma-\mathrm{CD}$ at $\mathrm{pH} 4.0$, which is close to the $\mathrm{p} K_{\mathrm{a}}$ of DSS, shows a decrease in fluorescence intensity of the LE band and a concomitant fluorescence enhancement from the CT band (Fig. 4b).

The $\mathrm{pH}$-dependent fluorescence titration of DSS at four different $\mathrm{pH}$ with and without $\gamma$-CD shows a visible change ( $c f$. Fig. 5a) in the fluorescence outcome due to the formation of $\gamma$-CD.(DSS $)_{2}$ (Fig. 3a). At a pH of around 3.0, the protonated

Table $2 \mathrm{pH}$-dependent binding constants of DSS with $\gamma$-CD and fluorescence enhancement and average fluorescence lifetime upon complexation

\begin{tabular}{lrllll}
\hline & $\begin{array}{l}K_{1}{ }^{a} \\
\left(\mathrm{M}^{-1}\right)\end{array}$ & $\begin{array}{l}K_{2}{ }^{a} \\
\left(\mathrm{M}^{-1}\right)\end{array}$ & $\begin{array}{l}K_{1} K_{2}{ }^{a} \\
\left(\mathrm{M}^{-2}\right)\end{array}$ & $\begin{array}{l}\text { Fluorescence } \\
\text { enhancement factor }^{c}\end{array}$ & $\begin{array}{l}\text { Average } \\
\text { lifetime }^{d}(\mathrm{~ns})\end{array}$ \\
\hline 2.0 & 12 & 5 & 60 & 1.59 & $5.5(1.5,2.0)^{e}$ \\
4.0 & 55 & 20 & 1100 & 20 & $5.9(1.6,1.9)^{e}$ \\
7.0 & 185 & 25 & $4625(2700)^{b}$ & 57 & $6.1(1.9)^{e}$ \\
9.0 & 155 & 15 & 2325 & 36 & $5.7(2.1)^{e}$
\end{tabular}

${ }^{a}$ Binding constant values are obtained from $1: 2$ fitting equation. ${ }^{47}$ ${ }^{b}$ Binding constant in $100 \mathrm{mM}$ phosphate buffer solution. ${ }^{c}$ Fluorescence enhancement factor was calculated from the intensity ratio of the $\gamma$-CD.(DSS) $)_{2}$ complex band at $c a .485 \mathrm{~nm}$ after full complexation (except for $\mathrm{pH} 2.0$ at $570 \mathrm{~nm}$ ) compared to that without $\gamma-\mathrm{CD}$, note a reduction of intensity at $380 \mathrm{~nm}$ at $\mathrm{pH} 2.0$ titration. ${ }^{d}$ Average fluorescence lifetime of DSS with $35 \mathrm{mM} \gamma$-CD. ${ }^{e}$ Values in parenthesis show average fluorescent lifetime of only DSS dye in an aqueous solution when monitored at $430 \mathrm{~nm}$ and $530 \mathrm{~nm}$ at $\mathrm{pH} 2.0$ and 4.0 and at $530 \mathrm{~nm}$ at $\mathrm{pH} 7.0$ and 9.0. 

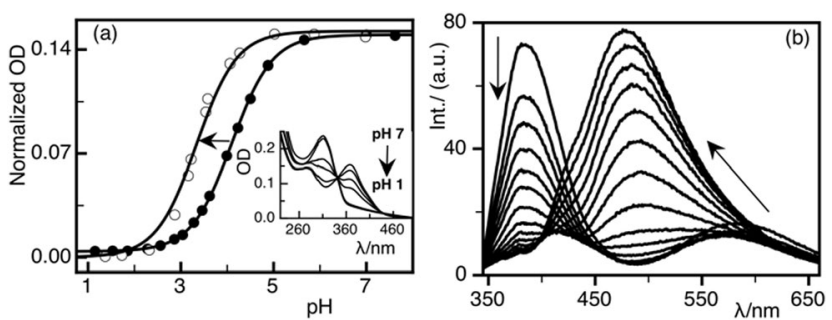

Fig. 4 (a) $\mathrm{pH}$ titration of DSS using the UV absorption of DSS without (closed circle) and with (open circle) $15 \mathrm{mM} \gamma$-CD at $340 \mathrm{~nm}$, showing ca. 1 unit $\mathrm{p} K_{\mathrm{a}}$ shift; inset shows the absorption sepctra of $\gamma$-CD.DSS at different $\mathrm{pH}$ using $15 \mathrm{mM}$ $\gamma-C D$, (b) binding titration of DSS with $\gamma-C D$ at $\mathrm{pH} 4.0$, a decrease at the $385 \mathrm{~nm}$ band and the commencement of the 1:2 complex band around $500 \mathrm{~nm}$.

form of DSS is weakly fluorescent upon excitation at $354 \mathrm{~nm}$ using a hand-held UV lamp, whereas at the same excitation, $\mathrm{pH} \gamma$-CD.(DSS $)_{2}$ shows a high fluorescence. Moreover, at $\mathrm{pH}$ 9.0, the deprotonated form of DSS exhibits a significant change in the fluorescence emission maxima after $\gamma$-CD complexation (see Fig. S1, ESI $\dagger$ ).

\subsection{Effect of $\gamma$-CD complexation on the fluorescence lifetime of DSS and $\mathrm{DSSH}^{+}$}

The fluorescence lifetime of DSS is known to be sensitive towards the solvent environment. ${ }^{40}$ In an aqueous solution, both DSS and $\mathrm{DSSH}^{+}$have approximately a fluorescence lifetime of $2.0 \mathrm{~ns}$, which can be related to strong intermolecular hydrogen bonding and associated with non-radiative deactivation pathways. ${ }^{52}$ In the present study, the fluorescence decay of DSS and its corresponding $\gamma-\mathrm{CD}$ complex were investigated in aqueous solutions at $\mathrm{pH} 2.0, \mathrm{pH} 4.0$, $\mathrm{pH}$ 7.0, and $\mathrm{pH}$ 9.0. The fluorescence lifetime of DSS and its $\gamma-\mathrm{CD}$ complex show bi-exponential decays (Fig. 5b, Table 2).

\subsection{Fluorescence anisotropy studies of DSS and its $\gamma$-CD complex}

Time-resolved fluorescence anisotropy measurements were carried out in aqueous solutions of DSS in the absence (Fig. 6a) and presence (Fig. 6b) of $15 \mathrm{mM} \gamma$-CD at $\mathrm{pH}$ 7.0. In both the cases, the anisotropy decays appeared to be mono-exponential in nature. A rotational correlation time $\left(\tau_{\mathrm{r}}\right)$ of $0.5 \mathrm{~ns}$ for DSS was obtained at pH 7.0. The anisotropy decay for $\gamma$-CD.(DSS $)_{2}$ was
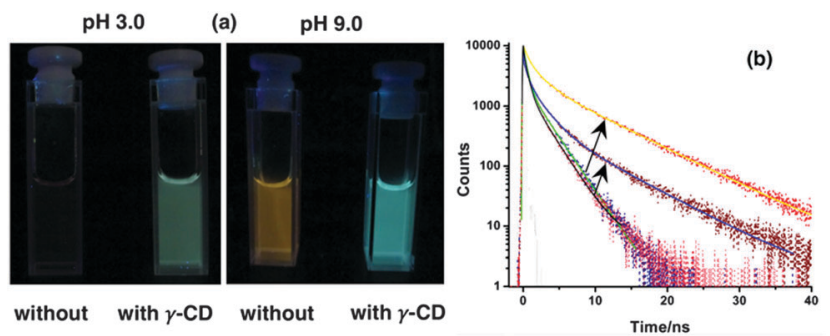

Fig. 5 (a) Digital images showing distinct fluorescent colour and intensity change of DSS $(10 \mu \mathrm{M})$ without and with $50 \mathrm{mM} \gamma-\mathrm{CD}$ at $\mathrm{pH} 3.0$ (left) and $\mathrm{pH} 9.0$ (right), (b) fluorescence lifetime enhancement (shown using arrow) of DSS $(5 \mu \mathrm{M})$ at $\mathrm{pH} 4.0$ (dark-brown) and $\mathrm{pH} 9.0$ (red) without and with $35 \mathrm{mM} \gamma-\mathrm{CD}$.
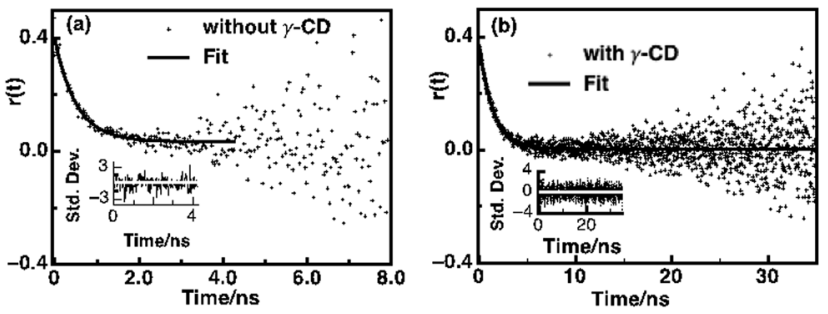

Fig. 6 Time-resolved anisotropy decay curves at $\mathrm{pH} 7.0$ in water at $25^{\circ} \mathrm{C}$ for (a) $5 \mu \mathrm{M}$ DSS without $\gamma-C D$ monitored at $580 \mathrm{~nm}$ and (b) the $5 \mu \mathrm{M}$ DSS and $15 \mathrm{mM} \gamma$-CD complex monitored at $480 \mathrm{~nm}$; inset shows residual of the fitting.

sufficiently slower compared to DSS, and it gave $\tau_{\mathrm{r}}$ values of about 1.3 ns. The initial anisotropy $\left(r_{0}\right)$ for both DSS and its corresponding $\gamma$-CD complex was also estimated to be considerably high ( $c a .0 .4)$. The reduced rotational diffusion times are fully consistent with the formation of inclusion complex. The rotational correlation time $\left(\tau_{\mathrm{r}}\right)$ can be related to its rotational diffusion coefficient and the viscosity of the surrounding environment according to the Stokes-Einstein relationship as follows:

$$
\tau=1 / 6 D_{\mathrm{r}}=\eta V / k T
$$

where $V$ is the hydrodynamic molecular volume of the complex, $\eta$ is the viscosity of the medium, $k$ is Boltzmann constant, and $T$ is the absolute temperature. For the inclusion complex between DSS and $\gamma-\mathrm{CD}$, a rough estimation of the hydrodynamic molecular volume can be obtained by considering the complex as an effective sphere and the hydrodynamic diameter similar to the outer diameter of a $\gamma$-CD molecule $(17.5 \AA)$ plus the volume corresponding to the part outside the $\gamma$-CD cavity. The volume $(V)$ of $\gamma$-CD can be calculated as $1690 \mathrm{~cm}^{3} \mathrm{~mol}^{-1}$. Thus, the total volume of the 1:2 complex can be calculated based on our theoretically calculated optimised structure (Fig. S2, ESI $\dagger$ ), where part of $N, N$-dimethyl aniline group is outside of the $\gamma$-CD cavity. For a single $N, N$-dimethyl aniline group, a total of $\sim 74 \mathrm{~cm}^{3} \mathrm{~mol}^{-1}$ $\left(\sim 43 \mathrm{~cm}^{3} \mathrm{~mol}^{-1}\right.$ for the benzene ring and $\sim 31 \mathrm{~cm}^{3} \mathrm{~mol}^{-1}$ for the $-\mathrm{N}\left(\mathrm{CH}_{3}\right)_{2}$ group) was estimated. ${ }^{53}$ Therefore, by considering a $\gamma$-CD.(DSS $)_{2}$ complex, a total volume of $\sim 1838 \mathrm{~cm}^{3} \mathrm{~mol}^{-1}$ ( $c f . \mathrm{ESI} \dagger$ for detail calculation) has been estimated. Therefore, from this volume and $\eta$ value of bulk water at $298 \mathrm{~K}$, we calculate $\tau_{\mathrm{r}}(0.72 \mathrm{~ns})$ of the complex, which is smaller presumably due to the underestimation of micro-viscosity as a result of hydrogen bonding network between the complex and solvent molecules. It is noteworthy that the slower rotational correlation time for the DSS suggests the formation of a tight inclusion complex, which appears to rotate as a whole in an aqueous solution, i.e. the independent motion of the dye inside the $\gamma$-CD cavity is highly restricted.

\subsection{Drug-binding affinity of $\gamma$-CD by fluorescence displacement assay}

Based on the modulation of optical properties of DSS upon complexation with the moderately large $\gamma$-CD cavity, we have designed a fluorescence turn-off-assay to evaluate the drug-binding propensity of its unique hydrophobic nanocavity, which can be 
(a)

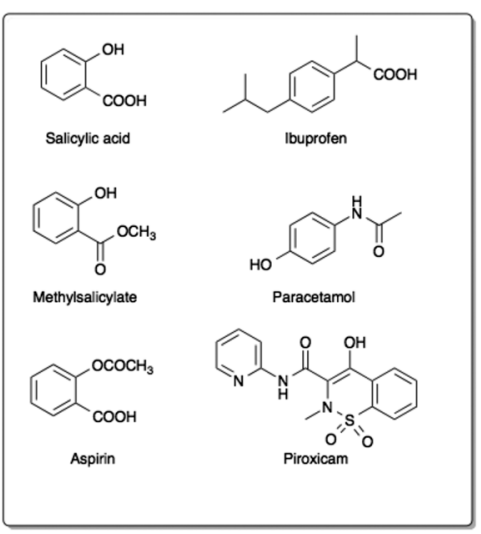

(b)

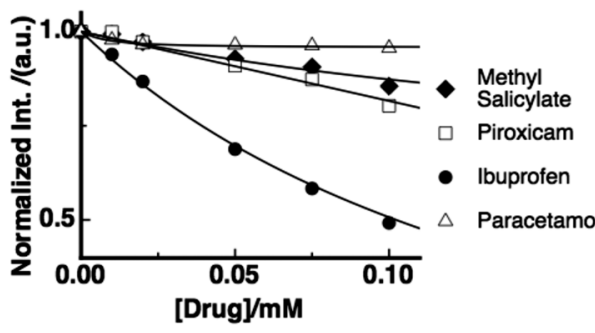

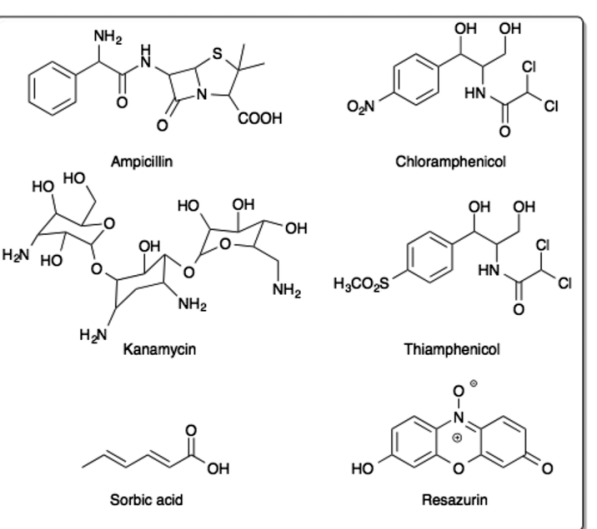

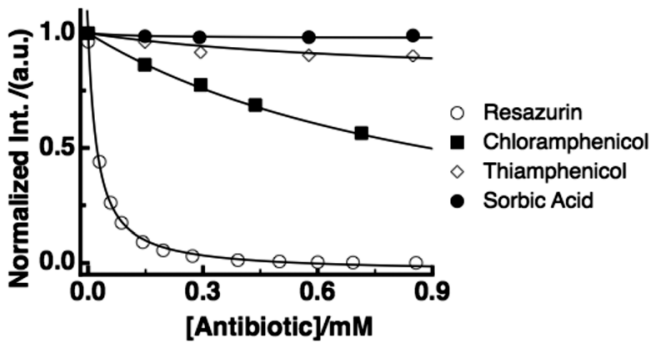

Fig. 7 (a) Structure of the drugs used to validate our principle; small molecule drugs (left) and antibiotic drugs (right); (b) relative change in fluorescence intensity and their corresponding fitted plot (solid line) upon displacement of DSS from the $\gamma$-CD cavity to quantify the binding affinity of small-molecule drug (left) and for antibiotic drugs (right).

used as an efficient carrier of sparingly water-soluble drugs to enhance their bioavailability. Twelve structurally different commodity drugs (viz. salicylic acid, methyl salicylate, aspirin, ibuprofen, piroxicam, paracetamol, ampicillin, kanamycin, chloramphenicol, thiamphenicol, sorbic acid and resazurin, cf. Fig. 7a) were selected to assess the complexation with the $\gamma$-CD cavity.

A $\gamma$-CD.(DSS $)_{2}$ fluorescent complex was pre-formed at $\mathrm{pH} 7.4$ using $5 \mu \mathrm{M}$ DSS and $5 \mathrm{mM} \gamma$-CD to obtain a significant effect of fluorescence enhancement. A binding titration of DSS with $\gamma$-CD in a phosphate buffer solution (100 mM, pH 7.4) was performed to test the suitability of the assay in physiological condition. We observed a significant fluorescence enhancement (Fig. S3, ESI $\dagger$ ) even in a $100 \mathrm{mM}$ PBS solution, which suggests a negligible competition from phosphate ions. Because the guest exchange equilibria between the guest and host are dynamic and rapid (nanosecond to millisecond) in nature and allows performing a displacement assay using a high concentration of host molecule. ${ }^{30}$ Subsequently, a gradual addition of drug solution, which was prepared in the same $\gamma$-CD.(DSS $)_{2}$ solution to avoid dilution, was performed to displace the fluorescent dye from the $\gamma$-CD cavity. Indeed, such displacement causes a gradual loss in fluorescence intensity due to the relocation of DSS dye into the aqueous solution. Such a decrease in fluorescence is an indirect indication of the binding strength of drug molecules. The binding affinity of small-molecule drugs and relatively larger antibiotic drugs with $\gamma$-CD (Fig. 7b, Table 3) was calculated by fitting the data ( $c f$. experimental section) for determining the change in fluorescence in terms of drug concentration.
Among the studied small-molecule drugs, ibuprofen shows the highest binding affinity towards $\gamma$-CD cavity followed by methyl salicylate, piroxicam and others. On the other hand, resazurin shows the highest affinity (independently measured by UV and fluorescence titration; as shown in Fig. S4 (ESI $\dagger$ ) and UV-Vis spectra of the drugs are provided in Fig. S5, ESI $\dagger$ ) among the antibiotic drugs followed by chloramphenicol and others. The binding constant of the drugs with $\gamma$-CD were calculated from the fitting (Table 3). The binding constant of the encapsulated drug within $\gamma$-CD is really important for their in vivo implication.

Table 3 Binding constant of drugs with $5 \mathrm{mM} \gamma$-CD at pH ca. 7.0 determined using $1: 1$ and $1: 2$ binding equations $s^{a, b}$

\begin{tabular}{lll}
\hline Entry & Drugs & Binding constant $\left(\mathrm{M}^{-1}\right)$ \\
\hline 1 & Ibuprofen & 5400 \\
2 & Piroxicam & 560 \\
3 & Salicyclic acid & $<20$ \\
4 & Methyl salicylate & 2400 \\
5 & Aspirin & $<10$ \\
6 & Paracetamol & $<10$ \\
7 & Ampicillin & $<50$ \\
8 & Kanamycin & $<10$ \\
9 & Chloramphenicol & 970 \\
10 & Resazurin & 48000 \\
11 & Thiamphenicol & 140 \\
12 & Sorbic acid & $<20$
\end{tabular}

${ }^{a}$ Drug binding constants were evaluated using a $1: 2$ equation for smallmolecules and a $1: 1$ equation for large antibiotic molecules. ${ }^{b}$ DSS binding with $\gamma$-CD is a dynamic equilibrium process and because the binding constant is related to the rates $\left(K_{1} K_{2}=k_{\text {on }} / k_{\text {off }}\right)$, the displacement of DSS from $\gamma$-CD can be realised as a dynamic process as well. 
A higher binding strength ( ca. $10^{4}-10^{5} \mathrm{M}^{-1}$ ) of the drug. $\mathrm{CD}$ complex will provide appreciable bioavailability, as reviewed comprehensively. ${ }^{54}$ Fluorescent-based, easy-to-perform quick assays can be useful to other class of drugs as well as for improving their chemical and physical properties to enhance bioavailability for drug delivery application.

\section{Conclusions}

In conclusion, a $\gamma$-CD.(DSS) $)_{2}$ complex was characterised using absorption, steady-state and time-resolved fluorescence spectroscopy and anisotropy measurements. The pH-dependent binding affinity of DSS with $\gamma$-CD shows preferential binding of the neutral form over the protonated form. Furthermore, a simple fluorescence displacement assay was developed to validate the drug-binding ability of the $\gamma$-CD cavity for small-molecule drugs and antibiotic drugs. This assay is operable under physiological conditions and is useful for any hydrophobic, neutral and negatively charged drugs. Our method will allow pharmaceutical industries to quickly assess and analyse the suitability of CD for drug formulation application. The same assay can be performed to test the thermal and chemical stability of the formulated drug in a high-throughput fashion. Such fluorescence-based displacement assays will allow us to quickly validate the suitability of $\gamma$-CD as a drug carrier for sparingly water-soluble drugs.

\section{Acknowledgements}

We would like to thank the Department of Science and Technology (DST), India for the research grant (SERB/CS-253/2013) and Indian Institute of Science Education and Research Bhopal (IISER Bhopal) for the infrastructure and initial financial support. KP thanks CSIR and SM thanks UGC for their doctoral fellowship.

\section{References}

1 K. Uekama, F. Hirayama and T. Irie, Chem. Rev., 1998, 98, 2045-2076.

2 K. Uekama, Adv. Drug Delivery Rev., 1999, 36, 1-2.

3 L. Szente and J. Szeman, Anal. Chem., 2013, 85, 8024-8030.

4 D. H. Macartney, Isr. J. Chem., 2011, 51, 600-615.

5 R. N. Dsouza, U. Pischel and W. M. Nau, Chem. Rev., 2011, 111, 7941-7980.

6 H. Bakirci, A. L. Koner and W. M. Nau, Chem. Commun., 2005, 5411-5413.

7 R. B. Wang, L. Yuan and D. H. Macartney, Chem. Commun., 2005, 5867-5869.

8 R. K. Gilpin and C. S. Gilpin, Anal. Chem., 2007, 79, 4275-4293.

9 K. S. S. P. Rao, S. M. Hubig, J. N. Moorthy and J. K. Kochi, J. Org. Chem., 1999, 64, 8098-8104.

10 D. J. Singhavi, S. Khan and P. G. Yeole, J. Mater. Sci.: Mater. Med., 2013, 24, 941-949.

11 N. Saleh, A. L. Koner and W. M. Nau, Angew. Chem., Int. Ed., 2008, 47, 5398-5401.
12 W. T. Duan, R. Liu and A. Sen, J. Am. Chem. Soc., 2013, 135, 1280-1283.

13 A. L. Koner, I. Ghosh, N. Saleh and W. M. Nau, Can. J. Chem., 2011, 89, 139-147.

14 I. Ghosh and W. M. Nau, Adv. Drug Delivery Rev., 2012, 64, 764-783.

15 J. M. Benito, M. Gomez-Garcia, C. O. Mellet, I. Baussanne, J. Defaye and J. M. G. Fernandez, J. Am. Chem. Soc., 2004, 126, 10355-10363.

16 D. Duchene, G. Ponchel and D. Wouessidjewe, Adv. Drug Delivery Rev., 1999, 36, 29-40.

17 Y. Yang, Y.-M. Zhang, Y. Chen, J.-T. Chen and Y. Liu, J. Med. Chem., 2013, 56, 9725-9736.

18 J. Szejtli, Chem. Rev., 1998, 98, 1743-1754.

19 N. J. Turro, T. Okubo and C. J. Chung, J. Am. Chem. Soc., 1982, 104, 3954-3957.

20 A. L. Koner and W. M. Nau, Supramol. Chem., 2007, 19, 55-66.

21 H. Bakirci and W. M. Nau, Adv. Funct. Mater., 2006, 16, 237-242.

22 M. E. Brewster and T. Loftsson, Adv. Drug Delivery Rev., 2007, 59, 645-666.

23 N. Schaschke, S. Fiori, E. Weyher, C. Escrieut, D. Fourmy, G. Muller and L. Moroder, J. Am. Chem. Soc., 1998, 120, 7030-7038.

24 V. Ramamurthy and D. F. Eaton, Acc. Chem. Res., 1988, 21, 300-306.

25 R. Breslow, Acc. Chem. Res., 1991, 24, 159-164.

26 A. Challa, A. Ahuja, J. Ali and R. K. Khar, AAPS Pharma. Sci. Tech., 2005, 6, E329-E357.

27 A. Chaudhury, U. Nagaich, N. Gulati, V. K. Sharma and R. L. Khosa, J. Adv. Pharm. Educ. Res., 2012, 2, 32-67.

28 T. Loftsson and M. Brewster, J. Pharm. Sci., 1996, 85, 1017-1025.

29 P. Mura, G. P. Bettinetti, A. Manderioli, M. T. Faucci, G. Bramanti and M. Sorrenti, Int. J. Pharm., 1998, 166, 189-203.

30 A. Hennig, H. Bakirci and W. M. Nau, Nat. Methods, 2007, 4, 629-632.

31 D. M. Bailey, A. Hennig, V. D. Uzunova and W. M. Nau, Chem. - Eur. J., 2008, 14, 6069-6077.

32 S. L. Wiskur, H. Ait-Haddou, J. J. Lavigne and E. V. Anslyn, Acc. Chem. Res., 2001, 34, 963-972.

33 Z. Diwu, Y. X. Lu, C. L. Zhang, D. H. Klaubert and R. P. Haugland, Photochem. Photobiol., 1997, 66, 424-431.

34 Z. J. Diwu, C. L. Zhang, D. H. Klaubert and R. P. Haugland, J. Photochem. Photobiol., A, 2000, 131, 95-100.

35 A. Nag, R. Dutta, N. Chattopadhyay and K. Bhattacharyya, Chem. Phys. Lett., 1989, 157, 83-86.

36 A. Nag and K. Bhattacharyya, J. Chem. Soc., Faraday Trans., 1990, 86, 53-54.

37 Z. R. Grabowski, K. Rotkiewicz and W. Rettig, Chem. Rev., 2003, 103, 3899-4031.

38 K. Bhattacharyya, J. Mol. Liq., 1993, 57, 115-125.

39 Q. Zhu, H. S. Yoon, P. B. Parikh, Y. T. Chang and S. Q. Yao, Tetrahedron Lett., 2002, 43, 5083-5086.

40 K. Pal, F. Chandra, S. Mallick and A. L. Koner, J. Photochem. Photobiol., A, 2015, 306, 47-54. 
41 R. N. Dsouza, A. Hennig and W. M. Nau, Chem. - Eur. J., 2012, 18, 3444-3459.

42 Y. Yang, H.-F. Yang, Y.-L. Liu, Z.-M. Liu, G.-L. Shen and R.-Q. Yu, Sens. Actuators, B, 2005, 106, 632-640.

43 P. Liu, S. Sun, X. Guo, X. Yang, J. Huang, K. Wang, Q. Wang, J. Liu and L. He, Anal. Chem., 2015, 87, 2665-2671.

44 H. Ikeda, M. Nakamura, N. Ise, N. Oguma, A. Nakamura, T. Ikeda, F. Toda and A. Ueno, J. Am. Chem. Soc., 1996, 118, 10980-10988.

45 L. Nogueiras-Nieto, E. Sobarzo-Sánchez, J. L. Gómez-Amoza and F. J. Otero-Espinar, Eur. J. Pharm. Biopharm., 2012, 80, 585-595.

46 H. Bakirci, A. L. Koner, T. Schwarzlose and W. M. Nau, Chem. - Eur. J., 2006, 12, 4799-4807.
47 H. Bakirci and W. M. Nau, J. Photochem. Photobiol., A, 2005, 173, 340-348.

48 D. E. Delley, A. J. Ellis, E. Freeman, J. Baerends and D. Post, Phys. Rev. B: Condens. Matter Mater. Phys., 1983, 27, 2132-2144.

49 J. P. Perdew, K. Burke and M. Ernzerhof, Phys. Rev. Lett., 1996, 77, 3865-3868.

50 W. Rettig, Angew. Chem., Int. Ed., 1986, 25, 971-988.

51 E. M. Kosower and H. Kanety, J. Am. Chem. Soc., 1983, 105, 6236-6243.

52 T. K. Mukherjee, P. Ahuja, A. L. Koner and A. Datta, J. Phys. Chem. B, 2005, 109, 12567-12573.

53 H. Bakirci, A. L. Koner and W. M. Nau, J. Org. Chem., 2005, 70, 9960-9966.

54 V. Stella and R. Rajewski, Pharm. Res., 1997, 14, 556-567. 